\title{
Some Properties for Fuzzy Differential Subordination Defined by Wanas Operator
}

\section{Şahsene Altınkaya ${ }^{1}$ and Abbas Kareem Wanas ${ }^{2}$}

${ }^{1}$ Department of Mathematics, Faculty of Arts and Science, Uludag University, Bursa, Turkey

e-mail: sahsene@uludag.edu.tr

${ }^{2}$ Department of Mathematics, College of Science, University of Al-Qadisiyah, Iraq e-mail: abbas.kareem.w@qu.edu.iq

\section{Abstract}

In this article, we establish some interesting geometric properties for fuzzy differential subordination associated with Wanas operator which defined in the open unit disk.

\section{Introduction}

Let the notation $\mathcal{H}(\mathcal{U})$ stand for the family of holomorphic functions in the unit disk $\mathcal{U}=\{z \in \mathbb{C}:|z|<1\}$. For $n \in \mathbb{N}$ and $a \in \mathbb{C}$, we indicate by

$$
\mathcal{H}[a, n]=\left\{f \in \mathcal{H}(\mathcal{U}): f(z)=a+a_{n} z^{n}+a_{n+1} z^{n+1}+\cdots, z \in \mathcal{U}\right\}
$$

and

$$
\mathcal{A}_{n}=\left\{f \in \mathcal{H}(\mathcal{U}): f(z)=z+a_{n+1} z^{n+1}+a_{n+2} z^{n+2}+\cdots, z \in \mathcal{U}\right\},
$$

with $\mathcal{A}_{1}=\mathcal{A}$.

Definition 1.1 [13]. Let $X$ be a non-empty set. An application $F: X \rightarrow[0,1]$ is called fuzzy subset. An alternate definition, more precise, would be the following:

Received: February 8, 2020; Accepted: March 28, 2020

2010 Mathematics Subject Classification: 30C45.

Keywords and phrases: fuzzy subordination, fuzzy set, fuzzy best dominant, Wanas operator.

Copyright (C) 2020 Şahsene Altınkaya and Abbas Kareem Wanas. This is an open access article distributed under the Creative Commons Attribution License, which permits unrestricted use, distribution, and reproduction in any medium, provided the original work is properly cited. 
A pair $\left(S, F_{S}\right)$, where $F_{S}: X \rightarrow[0,1]$ and $\operatorname{supp}\left(S, F_{S}\right)=\left\{x \in X: 0<F_{S}(x) \leq 1\right\}$ is called fuzzy subset. The function $F_{S}$ is called membership function of the fuzzy subset $\left(S, F_{S}\right)$.

Definition 1.2 [6]. Let two fuzzy subsets of $X,\left(M, F_{M}\right)$ and $\left(N, F_{N}\right)$. We say that the fuzzy subsets $M$ and $N$ are equal if and only if $F_{M}(x)=F_{N}(x), x \in X$ and we denote this by $\left(M, F_{M}\right)=\left(N, F_{N}\right)$. The fuzzy subset $\left(M, F_{M}\right)$ is contained in the fuzzy subset $\left(N, F_{N}\right)$ if and only if $F_{M}(x) \leq F_{N}(x), x \in X$ and we denote the inclusion relation by $\left(M, F_{M}\right) \subseteq\left(N, F_{N}\right)$.

Assume that $D$ is a set in $\mathbb{C}$ and $f, g$ are holomorphic functions. We indicate by

$$
f(D)=\operatorname{supp}\left(f(D), F_{f(D)}\right)=\left\{f(z): 0<F_{f(D)}(f(z)) \leq 1, z \in D\right\}
$$

and

$$
g(D)=\operatorname{supp}\left(g(D), F_{g(D)}\right)=\left\{g(z): 0<F_{g(D)}(g(z)) \leq 1, z \in D\right\} .
$$

Definition 1.3 [6]. Suppose that $D$ is a set in $\mathbb{C}, z_{0} \in D$ is a fixed point and let the functions $f, g \in \mathcal{H}(D)$. The function $f$ is named a fuzzy subordinate to $g$ and write $f \prec_{F} g$ or $f(z) \prec_{F} g(z)$ if satisfies the following:

(1) $f\left(z_{0}\right)=g\left(z_{0}\right)$,

(2) $F_{f(D)}(f(z)) \leq F_{g(D)}(g(z)), z \in D$.

Definition 1.4 [7]. Let $h$ be univalent in $\mathcal{U}$ and $\psi: \mathbb{C}^{3} \times \mathcal{U} \rightarrow \mathbb{C}$. If $\mathcal{P}$ is holomorphic in $\mathcal{U}$ satisfies the fuzzy differential subordination:

$$
F_{\psi\left(\mathbb{C}^{3} \times \mathcal{U}\right)}\left(\psi\left(\mathcal{P}(z), z \mathcal{P}^{\prime}(z), z^{2} \mathcal{P}^{\prime \prime}(z) ; z\right)\right) \leq F_{h(\mathcal{U})}(h(z)),
$$

i.e.,

$$
\psi\left(\mathcal{P}(z), z \mathcal{P}^{\prime}(z), z^{2} \mathcal{P}^{\prime \prime}(z) ; z\right) \prec_{F} h(z), z \in \mathcal{U},
$$

then $\mathcal{P}$ is called a fuzzy solution of the fuzzy differential subordination. The univalent function $q$ is called a fuzzy dominant of the fuzzy solutions of the fuzzy differential subordination, or more simple a fuzzy dominant, if $\mathcal{P}(z) \prec_{F} q(z), z \in \mathcal{U}$ for all $\mathcal{P}$ 
satisfying (1.1). A fuzzy dominant $\tilde{q}$ that satisfies $\tilde{q}(z) \prec_{F} q(z), z \in \mathcal{U}$ for all fuzzy dominant $q$ of (1.1) is said to be the fuzzy best dominant of (1.1).

For $\alpha \in \mathbb{R}, \beta \geq 0$ with $\alpha+\beta>0, m, \lambda \in \mathbb{N}_{0}=\mathbb{N} \bigcup\{0\}$ and $f \in \mathcal{A}$, we consider the differential operator $W_{\alpha, \beta}^{k, \lambda}: \mathcal{A} \rightarrow \mathcal{A}$, introduced by Wanas [11], where

$$
W_{\alpha, \beta}^{k, \lambda} f(z)=z+\sum_{n=2}^{\infty}\left[\sum_{m=1}^{k}\left(\begin{array}{l}
k \\
m
\end{array}\right)(-1)^{m+1}\left(\frac{\alpha^{m}+n \beta^{m}}{\alpha^{m}+\beta^{m}}\right)\right]^{\lambda} a_{n} z^{n} .
$$

By making use of (1.2), it is evident that

$$
\begin{aligned}
z\left(W_{\alpha, \beta}^{k, \lambda} f(z)\right)^{\prime}= & {\left[\sum_{m=1}^{k}\left(\begin{array}{l}
k \\
m
\end{array}\right)(-1)^{m+1}\left(\left(\frac{\alpha}{\beta}\right)^{m}+1\right)\right] W_{\alpha, \beta}^{k, \lambda+1} f(z) } \\
& -\left[\sum_{m=1}^{k}\left(\begin{array}{l}
k \\
m
\end{array}\right)(-1)^{m+1}\left(\frac{\alpha}{\beta}\right)^{m}\right] W_{\alpha, \beta}^{k, \lambda} f(z) .
\end{aligned}
$$

We will need the following lemmas in investigating our main results.

Lemma 1.1 [5]. Suppose that the convex function $h$ satisfies $h(0)=a$, let $\mu \in \mathbb{C}^{*}$ $=\mathbb{C} \backslash\{0\}$ such that $\operatorname{Re}(\mu) \geq 0$. If $\mathcal{P} \in \mathcal{H}[a, n]$ with $\mathcal{P}(0)=a$ and $\psi: \mathbb{C}^{2} \times \mathcal{U} \rightarrow \mathbb{C}$, $\psi\left(\mathcal{P}(z), z \mathcal{P}^{\prime}(z)\right)=\mathcal{P}(z)+\frac{1}{\mu} z \mathcal{P}^{\prime}(z)$ is holomorphic in $\mathcal{U}$, then

$$
F_{\psi\left(\mathbb{C}^{2} \times \mathcal{U}\right)}\left[\mathcal{P}(z)+\frac{1}{\mu} z \mathcal{P}^{\prime}(z)\right] \leq F_{h(\mathcal{U})} h(z)
$$

implies

$$
F_{\mathcal{P}(\mathcal{U})} \mathcal{P}(z) \leq F_{q(\mathcal{U})} q(z) \leq F_{h(\mathcal{U})} h(z), \quad z \in \mathcal{U},
$$

i.e.,

$$
\mathcal{P}(z) \prec_{F} q(z) \prec_{F} h(z),
$$

where

$$
q(z)=\frac{\mu}{n z^{\frac{\mu}{n}}} \int_{0}^{z} h(t) t^{\frac{\mu}{n}-1} d t
$$

is convex and is the fuzzy best dominant. 
Lemma 1.2 [5]. Suppose that $q$ is a convex function in $\mathcal{U}$, let $h(z)=q(z)+$ $n v z q^{\prime}(z), v>0$ and $n \in \mathbb{N}$. If $\mathcal{P} \in \mathcal{H}[q(0), n]$ and $\psi: \mathbb{C}^{2} \times \mathcal{U} \rightarrow \mathbb{C}, \psi\left(\mathcal{P}(z), z \mathcal{P}^{\prime}(z)\right)$ $=\mathcal{P}(z)+v z \mathcal{P}^{\prime}(z)$ is holomorphic in $\mathcal{U}$, then

$$
F_{\psi\left(\mathbb{C}^{2} \times \mathcal{U}\right)}\left[\mathcal{P}(z)+v z \mathcal{P}^{\prime}(z)\right] \leq F_{h(\mathcal{U})} h(z),
$$

implies

$$
F_{\mathcal{P}(\mathcal{U})} \mathcal{P}(z) \leq F_{q(\mathcal{U})} q(z), \quad z \in \mathcal{U}
$$

i.e.,

$$
\mathcal{P}(z) \prec_{F} q(z)
$$

and $q$ is the fuzzy best dominant.

Recently, Oros and Oros [7, 8], Lupaş [2-5], Haydar [1] and Wanas and Majeed [10, $11,12]$ have obtained fuzzy differential subordination results for certain classes of holomorphic functions.

\section{Main Results}

Theorem 2.1. Suppose that convex function $h$ satisfies $h(0)=1$. Let $f \in \mathcal{A}$ and

$$
\begin{aligned}
& \frac{1}{z}\left(\left[\sum_{m=1}^{k}\left(\begin{array}{l}
k \\
m
\end{array}\right)(-1)^{m+1}\left(\left(\frac{\alpha}{\beta}\right)^{m}+1\right)\right] W_{\alpha, \beta}^{k, \lambda+1} f(z)-\left[\sum_{m=1}^{k}\left(\begin{array}{l}
k \\
m
\end{array}\right)(-1)^{m+1}\left(\frac{\alpha}{\beta}\right)^{m}\right] W_{\alpha, \beta}^{k, \lambda} f(z)\right) \\
& +z\left(W_{\alpha, \beta}^{k, \lambda} f(z)\right)^{\prime \prime}
\end{aligned}
$$

is holomorphic in $\mathcal{U}$.

$$
\begin{aligned}
& \text { If } \\
& \qquad \begin{array}{l}
F_{\psi\left(\mathbb{C}^{2} \times \mathcal{U}\right)}\left[\frac { 1 } { z } \left(\left[\sum_{m=1}^{k}\left(\begin{array}{c}
k \\
m
\end{array}\right)(-1)^{m+1}\left(\left(\frac{\alpha}{\beta}\right)^{m}+1\right)\right] W_{\alpha, \beta}^{k, \lambda+1} f(z)\right.\right. \\
\left.\left.-\left[\sum_{m=1}^{k}\left(\begin{array}{c}
k \\
m
\end{array}\right)(-1)^{m+1}\left(\frac{\alpha}{\beta}\right)^{m}\right] W_{\alpha, \beta}^{k, \lambda} f(z)\right)+z\left(W_{\alpha, \beta}^{k, \lambda} f(z)\right)^{\prime \prime}\right] \leq F_{h(\mathcal{U})^{h}(z),}
\end{array}
\end{aligned}
$$


then

$$
F_{\left(W_{\alpha, \beta}^{k, \lambda} f\right)^{\prime}(\mathcal{U})}\left(\left(W_{\alpha, \beta}^{k, \lambda} f(z)\right)^{\prime}\right) \leq F_{q(\mathcal{U})} q(z) \leq F_{h(\mathcal{U})} h(z),
$$

i.e.

$$
\left(W_{\alpha, \beta}^{k, \lambda} f(z)\right)^{\prime} \prec_{F} q(z) \prec_{F} h(z),
$$

where $q(z)=\frac{1}{z} \int_{0}^{z} h(t)$ is convex and is the fuzzy best dominant.

Proof. Assume that

$$
\mathcal{P}(z)=\left(W_{\alpha, \beta}^{k, \lambda} f(z)\right)^{\prime}
$$

Then $\mathcal{P} \in \mathcal{H}[1,1]$ and $\mathcal{P}(0)=1$. Therefore, in view of (1.3) and (2.2), we have

$$
\begin{aligned}
\mathcal{P}(z)+z \mathcal{P}^{\prime}(z)= & 1+\sum_{n=2}^{\infty} n^{2}\left[\sum_{m=1}^{k}\left(\begin{array}{l}
k \\
m
\end{array}\right)(-1)^{m+1}\left(\frac{\alpha^{m}+n \beta^{m}}{\alpha^{m}+\beta^{m}}\right)\right]^{\lambda} a_{n} z^{n-1} \\
= & \sum_{m=1}^{k}\left(\begin{array}{l}
k \\
m
\end{array}\right)(-1)^{m+1}\left(\left(\frac{\alpha}{\beta}\right)^{m}+1\right) \\
& \times\left[1+\sum_{n=2}^{\infty}\left[\sum_{m=1}^{k}\left(\begin{array}{c}
k \\
m
\end{array}\right)(-1)^{m+1}\left(\frac{\alpha^{m}+n \beta^{m}}{\alpha^{m}+\beta^{m}}\right)\right]^{\lambda+1} a_{n} z^{n-1}\right] \\
& \left.-\sum_{m=1}^{k}\left(\begin{array}{c}
k \\
m
\end{array}\right)(-1)^{m+1}\left(\frac{\alpha}{\beta}\right)^{m}\right] \\
& \times\left[1+\sum_{n=2}^{\infty}\left[\sum_{m=1}^{k}\left(\begin{array}{c}
k \\
m
\end{array}\right)(-1)^{m+1}\left(\frac{\alpha^{m}+n \beta^{m}}{\alpha^{m}+\beta^{m}}\right)\right]^{\lambda} a_{n} z^{n-1}\right] \\
& +\sum_{n=2}^{\infty} n(n-1)\left[\sum_{m=1}^{k}\left(\begin{array}{l}
k \\
m
\end{array}\right)(-1)^{m+1}\left(\frac{\alpha^{m}+n \beta^{m}}{\alpha^{m}+\beta^{m}}\right)\right]^{\lambda} a_{n} z^{n-1}
\end{aligned}
$$




$$
\begin{aligned}
=\frac{1}{z}\left(\left[\sum_{m=1}^{k}\left(\begin{array}{l}
k \\
m
\end{array}\right)(-1)^{m+1}\left(\left(\frac{\alpha}{\beta}\right)^{m}+1\right)\right] W_{\alpha, \beta}^{k, \lambda+1} f(z)\right. \\
\left.-\left[\sum_{m=1}^{k}\left(\begin{array}{l}
k \\
m
\end{array}\right)(-1)^{m+1}\left(\frac{\alpha}{\beta}\right)^{m}\right] W_{\alpha, \beta}^{k, \lambda} f(z)\right)+z\left(W_{\alpha, \beta}^{k, \lambda} f(z)\right)^{\prime \prime} .
\end{aligned}
$$

According to (2.1) and (2.3), we deduce that

$$
F_{\psi\left(\mathbb{C}^{2} \times \mathcal{U}\right)}\left[\mathcal{P}(z)+z \mathcal{P}^{\prime}(z)\right] \leq F_{h(\mathcal{U})} h(z) .
$$

Thus applying Lemma 1.1 with $\mu=1$, we obtain

$$
F_{\mathcal{P}(\mathcal{U})} \mathcal{P}(z) \leq F_{q(\mathcal{U})} q(z) \leq F_{h(\mathcal{U})} h(z)
$$

From (2.2), we find that

$$
F_{\left(W_{\alpha, \beta}^{k, \lambda} f\right)^{\prime}(\mathcal{U})}\left(\left(W_{\alpha, \beta}^{k, \lambda} f(z)\right)^{\prime}\right) \leq F_{q(\mathcal{U})} q(z) \leq F_{h(\mathcal{U})} h(z),
$$

i.e.,

$$
\left(W_{\alpha, \beta}^{k, \lambda} f(z)\right)^{\prime} \prec_{F} q(z) \prec_{F} h(z),
$$

where $q(z)=\frac{1}{z} \int_{0}^{z} h(t) d t$ is convex and is the fuzzy best dominant.

Putting $\lambda=0$ and $h(z)=\frac{1+(2 \rho-1) z}{1+z}(0 \leq \rho<1)$ in Theorem 2.1, we obtain the following corollary:

Corollary 2.1. Let $f \in \mathcal{A}$ and $z f^{\prime \prime}(z)+f^{\prime}(z)$ is holomorphic in $\mathcal{U}$. If

$$
z f^{\prime \prime}(z)+f^{\prime}(z) \prec_{F} \frac{1+(2 p-1) z}{1+z},
$$

then

$$
f^{\prime}(z) \prec_{F} q(z) \prec_{F} \frac{1+(2 \rho-1) z}{1+z},
$$

where $q(z)=2 \rho-1+\frac{2(1-\rho)}{z} \ln (1+z)$ is convex and is the fuzzy best dominant. 
Theorem 2.2. Suppose that the convex function $h$ satisfies $h(0)=1$. Let $f \in \mathcal{A}$ and $\left(W_{\alpha, \beta}^{k, \lambda} f(z)\right)^{\prime}$ is holomorphic in $\mathcal{U}$. If

$$
F_{\psi\left(\mathbb{C}^{2} \times \mathcal{U}\right)}\left[\left(W_{\alpha, \beta}^{k, \lambda} f(z)\right)^{\prime}\right] \leq F_{h(\mathcal{U})} h(z),
$$

then

$$
F_{\left(W_{\alpha, \beta}^{k, \lambda} f\right)(\mathcal{U})}\left(\frac{W_{\alpha, \beta}^{k, \lambda} f(z)}{z}\right) \leq F_{q(\mathcal{U})} q(z) \leq F_{h(\mathcal{U})} h(z),
$$

i.e.,

$$
\frac{W_{\alpha, \beta}^{k, \lambda} f(z)}{z} \prec_{F} q(z) \prec_{F} h(z),
$$

where $q(z)=\frac{1}{z} \int_{0}^{z} h(t) d t$ is convex and is the fuzzy best dominant.

Proof. Assume that

$$
\mathcal{P}(z)=\frac{W_{\alpha, \beta}^{k, \lambda} f(z)}{z} .
$$

It is clear that $\mathcal{P} \in \mathcal{H}[1,1]$ and $\mathcal{P}(0)=1$.

We find

$$
\mathcal{P}(z)+z \mathcal{P}^{\prime}(z)=\left(W_{\alpha, \beta}^{k, \lambda} f(z)\right)^{\prime}
$$

In view of (2.6), the fuzzy differential subordination (2.4) becomes

$$
F_{\psi\left(\mathbb{C}^{2} \times \mathcal{U}\right)}\left[\mathcal{P}(z)+z \mathcal{P}^{\prime}(z)\right] \leq F_{h(\mathcal{U})} h(z) .
$$

Thus applying Lemma 1.1 with $\mu=1$, we obtain

$$
F_{\mathcal{P}(\mathcal{U})} \mathcal{P}(z) \leq F_{q(\mathcal{U})} q(z) \leq F_{h(\mathcal{U})} h(z) .
$$

From (2.5), we get

$$
F_{\left(W_{\alpha, \beta}^{k, \lambda} f\right)(\mathcal{U})}\left(\frac{W_{\alpha, \beta}^{k, \lambda} f(z)}{z}\right) \leq F_{q(\mathcal{U})} q(z) \leq F_{h(\mathcal{U})} h(z),
$$


i.e.,

$$
\frac{W_{\alpha, \beta}^{k, \lambda} f(z)}{z} \prec_{F} q(z) \prec_{F} h(z),
$$

where $q(z)=\frac{1}{z} \int_{0}^{z} h(t) d t$ is convex and is the fuzzy best dominant.

Putting $\lambda=0$ and $h(z)=e^{b z},|b| \leq 1$ in Theorem 2.2, we obtain the following corollary:

Corollary 2.2. If $f \in \mathcal{A}, f^{\prime}(z)$ is holomorphic in $\mathcal{U}$ and $f^{\prime}(z) \prec_{F} e^{b z}$, then

$$
\frac{f(z)}{z} \prec_{F} q(z) \prec_{F} e^{b z},
$$

where $q(z)=\frac{e^{b z}-1}{b z}$ is convex and is the fuzzy best dominant.

Theorem 2.3. Suppose that $q$ is a convex function in $\mathcal{U}$ such that $q(0)=1$, $h(z)=q(z)+\left[\sum_{m=1}^{k}\left(\begin{array}{l}k \\ m\end{array}\right)(-1)^{m+1}\left(\frac{\beta^{m}}{\alpha^{m}+\beta^{m}}\right)\right] z q^{\prime}(z)$. Let $f \in \mathcal{A}$ and $\left(W_{\alpha, \beta}^{k, \lambda+1} f(z)\right)^{\prime}$ is holomorphic in $\mathcal{U}$. If

$$
F_{\psi\left(\mathbb{C}^{2} \times \mathcal{U}\right)}\left[\left(W_{\alpha, \beta}^{k, \lambda+1} f(z)\right)^{\prime}\right] \leq F_{h(\mathcal{U})} h(z),
$$

then

$$
F_{\left(W_{\alpha, \beta}^{k, \lambda} f\right)^{\prime}(\mathcal{U})}\left(\left(W_{\alpha, \beta}^{k, \lambda} f(z)\right)^{\prime}\right) \leq F_{q(\mathcal{U})} q(z),
$$

i.e.,

$$
\left(W_{\alpha, \beta}^{k, \lambda} f(z)\right)^{\prime} \prec_{F} q(z)
$$

and $q$ is fuzzy best dominant.

Proof. Assume that

$$
\mathcal{P}(z)=\left(W_{\alpha, \beta}^{k, \lambda} f(z)\right)^{\prime}
$$

It is clear that $p \in \mathcal{H}[1,1]$. 
By simple computations of (2.8), we find that

$$
\begin{aligned}
& \mathcal{P}(z)+\left[\sum_{m=1}^{k}\left(\begin{array}{l}
k \\
m
\end{array}\right)(-1)^{m+1}\left(\frac{\beta^{m}}{\alpha^{m}+\beta^{m}}\right)\right] z \mathcal{P}^{\prime}(z) \\
= & \left(W_{\alpha, \beta}^{k, \lambda} f(z)\right)^{\prime}+\left[\sum_{m=1}^{k}\left(\begin{array}{l}
k \\
m
\end{array}\right)(-1)^{m+1}\left(\frac{\beta^{m}}{\alpha^{m}+\beta^{m}}\right)\right] z\left(W_{\alpha, \beta}^{k, \lambda} f(z)\right)^{\prime \prime} .
\end{aligned}
$$

Using (1.3) and differentiating with respect to $z$, we obtain

$$
\left(W_{\alpha, \beta}^{k, \lambda+1} f(z)\right)^{\prime}=\left(W_{\alpha, \beta}^{k, \lambda} f(z)\right)^{\prime}+\left[\sum_{m=1}^{k}\left(\begin{array}{l}
k \\
m
\end{array}\right)(-1)^{m+1}\left(\frac{\beta^{m}}{\alpha^{m}+\beta^{m}}\right)\right] z\left(W_{\alpha, \beta}^{k, \lambda} f(z)\right)^{\prime \prime} .
$$

In the light of (2.9) and (2.10), (2.7) becomes

$$
F_{\psi\left(\mathbb{C}^{2} \times \mathcal{U}\right)}\left[\mathcal{P}(z)+\left[\sum_{m=1}^{k}\left(\begin{array}{l}
k \\
m
\end{array}\right)(-1)^{m+1}\left(\frac{\beta^{m}}{\alpha^{m}+\beta^{m}}\right)\right] z \mathcal{P}^{\prime}(z)\right] \leq F_{h(\mathcal{U})} h(z) .
$$

Thus applying Lemma 1.2 with $v=\left[\sum_{m=1}^{k}\left(\begin{array}{l}k \\ m\end{array}\right)(-1)^{m+1}\left(\frac{\beta^{m}}{\alpha^{m}+\beta^{m}}\right)\right]$, we obtain

$$
F_{\left(W_{\alpha, \beta}^{k, \lambda} f\right)^{\prime}(\mathcal{U})}\left(\left(W_{\alpha, \beta}^{k, \lambda} f(z)\right)^{\prime}\right) \leq F_{q(\mathcal{U})} q(z),
$$

i.e.,

$$
\left(W_{\alpha, \beta}^{k, \lambda} f(z)\right)^{\prime} \prec_{F} q(z)
$$

and $q$ is fuzzy best dominant.

Theorem 2.4. Suppose that $q$ is a convex function in $\mathcal{U}$ such that $q(0)=1$,

$$
\begin{gathered}
h(z)=q(z)+z q^{\prime}(z) \text {. Let } f \in \mathcal{A} \text { and }\left(\frac{z W_{\alpha, \beta}^{k, \lambda+1} f(z)}{W_{\alpha, \beta}^{k, \lambda} f(z)}\right)^{\prime} \text { is holomorphic in } \mathcal{U} \text {. If } \\
F_{\psi\left(\mathbb{C}^{2} \times \mathcal{U}\right)}\left[\left(\frac{z W_{\alpha, \beta}^{k, \lambda+1} f(z)}{W_{\alpha, \beta}^{k, \lambda} f(z)}\right)^{\prime}\right] \leq F_{h(\mathcal{U})^{h} h(z),}
\end{gathered}
$$


then

$$
\left.F_{\left(\frac{W_{\alpha, \beta}^{k, \lambda+1} f}{W_{\alpha, \beta}^{k, \lambda} f}\right.}\right)(\mathcal{U})\left(\frac{W_{\alpha, \beta}^{k, \lambda+1} f(z)}{W_{\alpha, \beta}^{k, \lambda} f(z)}\right) \leq F_{q(\mathcal{U})} q(z),
$$

i.e.,

$$
\frac{W_{\alpha, \beta}^{k, \lambda+1} f(z)}{W_{\alpha, \beta}^{k, \lambda} f(z)} \prec_{F} q(z)
$$

and $q$ is fuzzy best dominant.

Proof. Assume that

$$
\mathcal{P}(z)=\frac{W_{\alpha, \beta}^{k, \lambda+1} f(z)}{W_{\alpha, \beta}^{k, \lambda} f(z)}
$$

Therefore, we note that $\mathcal{P} \in \mathcal{H}[1,1]$.

Differentiating both sides of (2.12) with respect to $z$, it yields

$$
\mathcal{P}^{\prime}(z)=\frac{\left(W_{\alpha, \beta}^{k, \lambda+1} f(z)\right)^{\prime}}{W_{\alpha, \beta}^{k, \lambda} f(z)}-\mathcal{P}(z) \frac{\left(W_{\alpha, \beta}^{k, \lambda} f(z)\right)^{\prime}}{W_{\alpha, \beta}^{k, \lambda} f(z)} .
$$

Then

$$
\begin{aligned}
\mathcal{P}(z)+z \mathcal{P}^{\prime}(z) & =\frac{W_{\alpha, \beta}^{k, \lambda} f(z)\left(z\left(W_{\alpha, \beta}^{k, \lambda+1} f(z)\right)^{\prime}+W_{\alpha, \beta}^{k, \lambda+1} f(z)\right)-z W_{\alpha, \beta}^{k, \lambda+1} f(z)\left(W_{\alpha, \beta}^{k, \lambda} f(z)\right)^{\prime}}{\left(W_{\alpha, \beta}^{k, \lambda} f(z)\right)^{2}} \\
& =\left(\frac{z W_{\alpha, \beta}^{k, \lambda+1} f(z)}{W_{\alpha, \beta}^{k, \lambda} f(z)}\right)^{\prime} .
\end{aligned}
$$

Utilizing (2.13) in (2.11), we can get

$$
F_{\psi\left(\mathbb{C}^{2} \times \mathcal{U}\right)}\left[\mathcal{P}(z)+z \mathcal{P}^{\prime}(z)\right] \leq F_{h(\mathcal{U})} h(z) .
$$


Thus applying Lemma 1.2 with $v=1$, we obtain

$$
\left.F_{\left(\frac{W_{\alpha, \beta}^{k, \lambda+1} f}{W_{\alpha, \beta}^{k, \lambda} f}\right.}\right)(\mathcal{U})\left(\frac{W_{\alpha, \beta}^{k, \lambda+1} f(z)}{W_{\alpha, \beta}^{k, \lambda} f(z)}\right) \leq F_{q(\mathcal{U})} q(z),
$$

i.e.,

$$
\frac{W_{\alpha, \beta}^{k, \lambda+1} f(z)}{W_{\alpha, \beta}^{k, \lambda} f(z)} \prec_{F} q(z)
$$

and $q$ is fuzzy best dominant.

\section{References}

[1] A. Haydar Eş, On fuzzy differential subordination, Math. Morav. 19(1) (2015), 123-129. https://doi.org/10.5937/MatMor1501123H

[2] A. Alb Lupaş, A note on special fuzzy differential subordinations using multiplier transformation, An. Univ. Oradea, Fasc. Mat. XXIII(2) (2016), 183-191.

[3] A. Alb Lupaş, On special fuzzy differential subordinations using generalized Sălăgean operator and Ruscheweyh derivative, J. Adv. Appl. Comp. Math. 4 (2017), 26-34. https://doi.org/10.15377/2409-5761.2017.04.5

[4] A. Alb Lupaş, On special fuzzy differential subordinations using multiplier transformation, J. Comput. Anal. Appl. 23(6) (2017), 1029-1035.

[5] A. Alb Lupaş, A note on special fuzzy differential subordinations using multiplier transformation and Ruschewehy derivative, J. Comput. Anal. Appl. 25(6) (2018), 11161124 .

[6] G. I. Oros and Gh. Oros, The notion of subordination in fuzzy set theory, Gen. Math. 19(4) (2011), 97-103.

[7] G. I. Oros and Gh. Oros, Fuzzy differential subordination, Acta Univ. Apulensis 30 (2012), 55-64.

[8] G. I. Oros and Gh. Oros, Dominants and best dominants in fuzzy differential subordinations, Stud. Univ Babeş-Bolyai Math. 57(2) (2012), 239-248.

[9] A. K. Wanas, New differential operator for holomorphic functions, Earthline J. Math. Sci. 2(2) (2019), 527-537. https://doi.org/10.34198/ejms.2219.527537 
[10] A. K. Wanas and A. H. Majeed, Fuzzy differential subordinations for prestarlike functions of complex order and some applications, Far East J. Math. Sci. 102(8) (2017), 17771788. https://doi.org/10.17654/MS102081777

[11] A. K. Wanas and A. H. Majeed, Fuzzy differential subordination properties of analytic functions involving generalized differential operator, Sci. Int. (Lahore) 30(2) (2018), 297302.

[12] A. K. Wanas and A. H. Majeed, Fuzzy subordination results for fractional integral associated with generalized Mittag-Leffler function, Eng. Math. Lett. 2019 (2019), Art. ID 10, pp. 1-13.

[13] L. A. Zadeh, Fuzzy sets, Information and Control 8 (1965), 338-353. https://doi.org/10.1016/S0019-9958(65)90241-X 